\title{
March Updates: People and Places
}

\section{Kovalenko medal awarded}

The 2018 Jessie Stevenson Kovalenko Medal, awarded every two years by the US National Academy of Sciences in recognition of outstanding research in medical sciences, has been given to cancer immunologist James Allison at the University of Texas MD Anderson Cancer Center. Allison's basic and preclinical work on the molecules involved in $\mathrm{T}$ cell function gave rise to the field of immune checkpoint therapies that target the immune system in cancer patients, rather than their tumors. Subsequently, Allison worked with pharmaceutical companies to develop the monoclonal antibody drug ipilimumab to treat stage- 4 melanoma; it was approved by the FDA in 2011.

Allison continues to investigate the basics of the immune system as well ways to improve immunotherapies in cancer patients. He will receive the Kovalenko medal during the Academy's Annual Meeting on April 29, along with a $\$ 25,000$ prize and a \$50,000 grant in support his research.

\section{Priming veterinary vaccines}

The International Veterinary Vaccinology Network, a network formed last December with a $£ 2.1 \mathrm{M}$ award from the Medical Research Council and the Biotechnology and Biological Sciences Research Council to help researchers in the UK and low-andmiddle income countries develop livestock vaccines, has announced the first recipients of its pump-priming grants.
Three projects were funded in this initial round. The first, to an international group working in Canada, the UK, and Vietnam, will study the immune system of farmed Nile tilapia and develop improved diseasemanagement strategies for aquaculture systems. The second project will attempt to create a low-cost, thermostable vaccine for Rift Valley fever for veterinary use. The third project will work to develop a single dose vaccine against Taenia solium, a tapeworm that causes neurocysticercosis in people and is carried by pigs.

The next round of pump-priming grant awardees will be announced at the Network's first scientific meeting in March 2018.

\section{Professional Excellence Awards The Association of American Veterinary Medical Colleges (AAVMC) has announced the recipients of the 2018 Professional Excellence Awards, to be presented during the AAVMC's Annual Conference and Assembly in March. Jennifer Hodgson, associate dean of the Virginia-Maryland College of Veterinary Medicine will receive the AAVMC Distinguished Teacher Award. Cornell University College of Veterinary Medicine virology professor Colin Parrish will receive the AAVMC Excellence in Research Award. James Roth from the Iowa State University's College of Veterinary Medicine will receive the Senator John Melcher, DVM Leadership in Public Policy Award. And Norman Williamson from Massey University in New Zealand}

\section{Careers update}

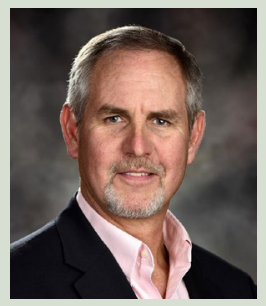

Brian Berridge will replace John Bucher as Associate Director of the National Toxicology Program (NTP). Berridge received his Doctor of Veterinary Medicine degree from Oklahoma State University and also completed a PhD in pathology in the Department of Veterinary Pathobiology at Texas A\&M University. For the past five years, he has directed the worldwide animal research strategy at GlaxoSmithKline. He has also advised the NTP previously as a member of the Scientific Advisory Committee on Alternative Toxicological Methods.

The NTP, part of the NIH National Institute of Environmental Health Sciences, coordinates toxicology research across nine federal agencies. In his role as Associate Director, Berridge will oversee toxicology studies as well as the production of NTP products, like the Report on Carcinogens.

Commenting in a press release, NTP Director Linda Birnbaum said, "We are thrilled that Dr. Berridge is bringing his expertise to environmental health, including experience with both traditional and novel toxicological methods."

will receive the Billy E. Hooper Award for Distinguished Service.

AAVMC CEO Andrew Maccabe commented in a press release, "These individuals represent the very best of what thousands of outstanding educators and researchers bring to their work every day in our member institutions... We're pleased to be able to recognize them for their outstanding achievements and contributions."

\section{JAX promotion}

LuAnn Ballesteros has been promoted to vice president, external and government affairs, at the Jackson Laboratory, a nonprofit research institution based in Bar Harbor, ME. Ballesteros joined the company as a senior development officer in 2005 and has director government affairs since 2011. She commented in a press release, "The Jackson Laboratory plays a critical role in the global biomedical research community and has far-reaching economic impact in Maine, Connecticut and California. I am thrilled to have the opportunity to advance a policy agenda that supports JAX's mission, grows the research sector and increases economic activity in the states where we have facilities."

\section{Immunology at Charles River}

Charles River Laboratories has announced its acquisition of Bristol-based KWS BioTest for $£ 15$ million in cash. An additional payment of $£ 3$ million based on future performance was also included in the transaction. KWS BioTest is a contract research organization that specializes in in vitro and in vivo testing services for immune-oncology and inflammatory and infectious diseases.

James Foster, President and CEO of Charles River, commented in a press release, "The addition of KWS strategically expands Charles River's existing discovery capabilities in the field of immunology, which is critical given the importance of new therapies which harness the human immune system. In addition to enhancing our position as the premier single-source provider for a broad portfolio of discovery services, KWS increases our ability to support clients' earlystage drug research in critical therapeutic areas, and expands our geographic footprint in the United Kingdom." 